\title{
Path loss Optimization in WIMAX Network using Genetic Algorithm
}

\author{
Shahad Nafea ${ }^{1}$, Ekhlas Kadum Hamza ${ }^{2}$ \\ Control \& System engineering department, University of Technology, Baghdad, Iraq. \\ shahadnafaa_1994@yahoo.com,100374@uotechnology.edu.iq
}

\begin{abstract}
The most necessary factors effect on the standard of broadband access services in mobile systems are WIMAX signal throughput and area coverage range. The environment controls are based on the sign power of any radio communication system. The sign power in any base station site relies on the space between the transmitter and the receiver, carrier frequency for the transmitter and the receiver along on the path loss. One of the most generally utilized experimental model to predict the path loss is the COST-231 Hata model. In this paper, the path loss rates have calculated based on the WIMAX criterion frequency at an area with $3.5 \mathrm{GHz}$, that constant distances $(7 \mathrm{~km})$ of transmitting base station into receive base station in urban, suburban environments. The execution of the COST-231 Hata paradigm and optimized paradigm are rated over the path loss. This model is optimized using Genetic Algorithm Technique using a computational tool MATLAB. Path loss results acquired show that the optimized paradigm rates a little higher than rates standard. The distance $(7 \mathrm{~km})$ is good because of achieving the lowest value for path loss.
\end{abstract}

Index Terms-WIMAX, COST-231 Hata, Genetic Algorithm

\section{Introduction}

Leading roles at the execution of communication systems are played by wireless environment. WIMAX (Worldwide Interoperability for Microwave Access) network has been become fundamental by the field of wireless broadband communications. WIMAX is designed to meet the need for very high-speed extensivearea Internet arrival pliable way and as well worked at a soft-price. WIMAX network was styled to get aloft-speed data and aloft stimulate invention in serving, satisfying, and modern mobile systems. WIMAX could typically prop data average of 500kbps to 2Mbps. WIMAX technology is based on (IEEE 802.16) standards. There are two central sorts of WIMAX, fixed WIMAX (IEEE 802.16d-2004), and mobile WIMAX (IEEE 802.16e-2005). Fixed WIMAX operates in rang frequency bands $2.5 \mathrm{GHz}, 3.5 \mathrm{GHz}$ (license) and $5.8 \mathrm{GHz}$ (license-free). Mobile WIMAX (802.16e) operates (2-6) GHz frequency bands. The Mobile WIMAX (IEEE 802.16e) was advanced for supply area coverage by the best data average. IEEE 802.16e releases (2005) support both the Frequency Division Duplexing (FDD) and the Time Division Duplexing (TDD). The channel bandwidths of 1.25 to $20 \mathrm{MHz}$ is supported by IEEE 802.16e [1] .WIMAX supports urban, suburban and rural broadband coverage. WIMAX coverage is based on path loss. The concept of path loss is a fundamental concept in planning any type of radio communications link. The difference between transmitted and received power is defined as path loss symbolized in $(\mathrm{dB})$. A connection link is strong or else whereby to near the system into fail could important at several states. Propagation models traditionally focus on the prediction of signal strength at the receiver, and distance (d) between the transmitter and the receiver that plays the most critical role on the received signal strength. 
The literature reviews of some of the published papers have been done, which is briefly described below: M. Alshami, et., al., (2011) [2] presented this research for all the PL models of WIMAX base station (BS) to WIMAX mobile station (MS) to different paradigms based on the frequency set at the band from (26) $\mathrm{GHz}$ with a constant distance $(5 \mathrm{~km})$ of transmitting to the receiving at urban, suburban and rural environments. M.Dalela, et al., (2012) [3] used a hybrid optimization to optimize the parameters of the COST-231Hata propagation model and validated frequency at $900 \mathrm{MHz}$ in the suburban region. $\mathbf{O}$. Banimelhem, et., al.,(2015) [4] optimized Hata model using genetic algorithm and swarm optimization which was validated by measurements collected from experimental sites. In this paper, we use the wide propagation model is COST-231 Hata and optimize through genetic algorithm. The optimize steps to get the best possible values by using path loss coefficients i.e., carrier frequency, base station antenna height, mobile station antenna height and the distance between BS and MS. The rest of the paper is structure as follows. Sections 2 describes propagation model. Section 3 discusses optimization in genetic algorithm. Section 4 presents results and discussion. Conclusions are drawn in section 5.

\section{Propagation Model}

At mobile networks, the signals are transmitted from the BSs to the MSs then to vice versa. Radio channels are modeled by five components, which are: the transmit antenna, path loss, fast fading, shadow fading, and the receive antenna. Path loss models versus distances, path loss, transmitting power and receiving power [5]. Link margin is a parameter utilized for gauge what near a link is of failure. The difference amidst of the system gains and the system losses are defined by Link margin. The successful connection happens where a link margin is higher than zero [6]. Therefore, the equation illustrates the mathematical relationship between transmit power, receiver sensitivity, antenna gain and path loss. The attenuation from a median between a transmit antenna and a receive antenna is called path loss as shown in Figure (1) [7].

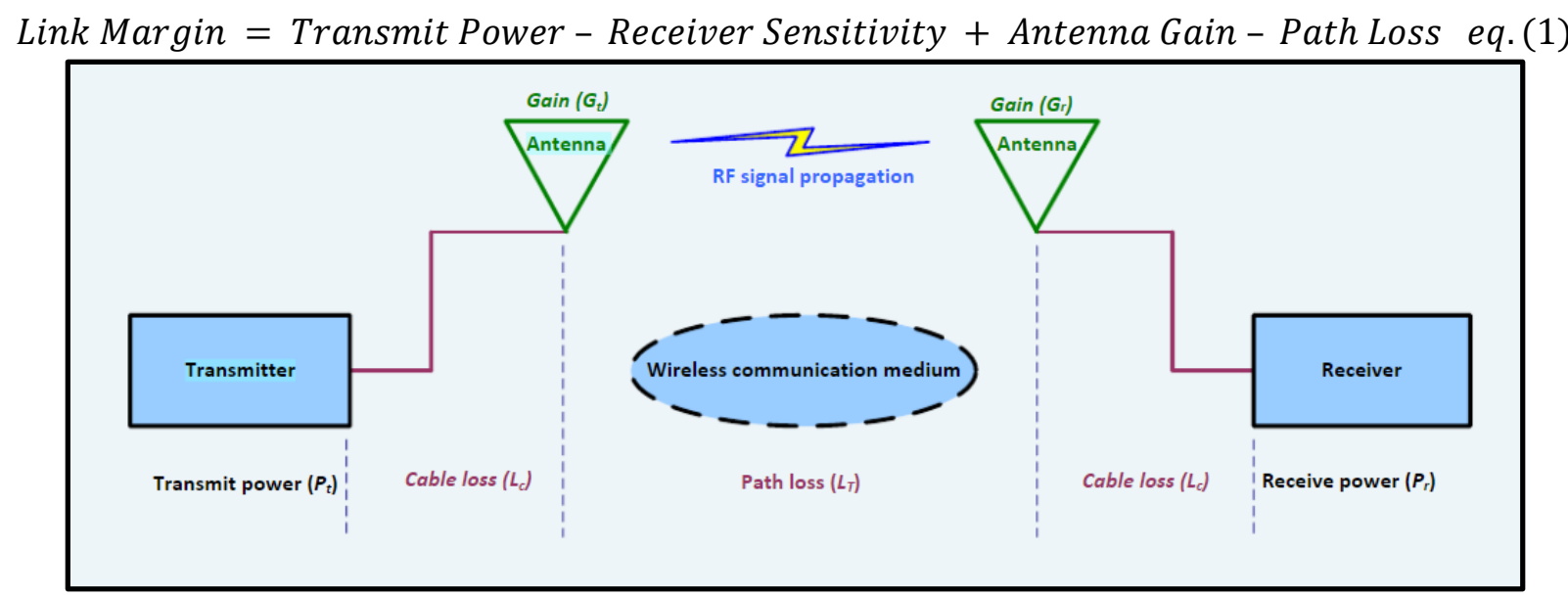

FiguRE (1): A TYPICAL MOBILE WIRELESS COMMUNICATION SYSTEM [7].

For simulations purposes the path loss is typically calculated based on models. The Path loss paradigms utilize a collection from athletic equations and algorithms for the prognosticate at path loss rates [7].The Path loss is effected by other things, the height from a transmitter base station and receiver antennas distance; however there is the Line of Sight (LOS) or the Non Line of Sight (NLOS). Figure (2) illustrates these parameters in the system. We will primarily carry out the impact of carrier frequency $\mathrm{f}(\mathrm{MHz})$ and 
distance $\mathrm{d}(\mathrm{km})$ on the path loss. In addition, the sensitivity analysis, which provides critical parameters in the system with the most impact on $d$, is incorporated for the system design and planning purpose [7].

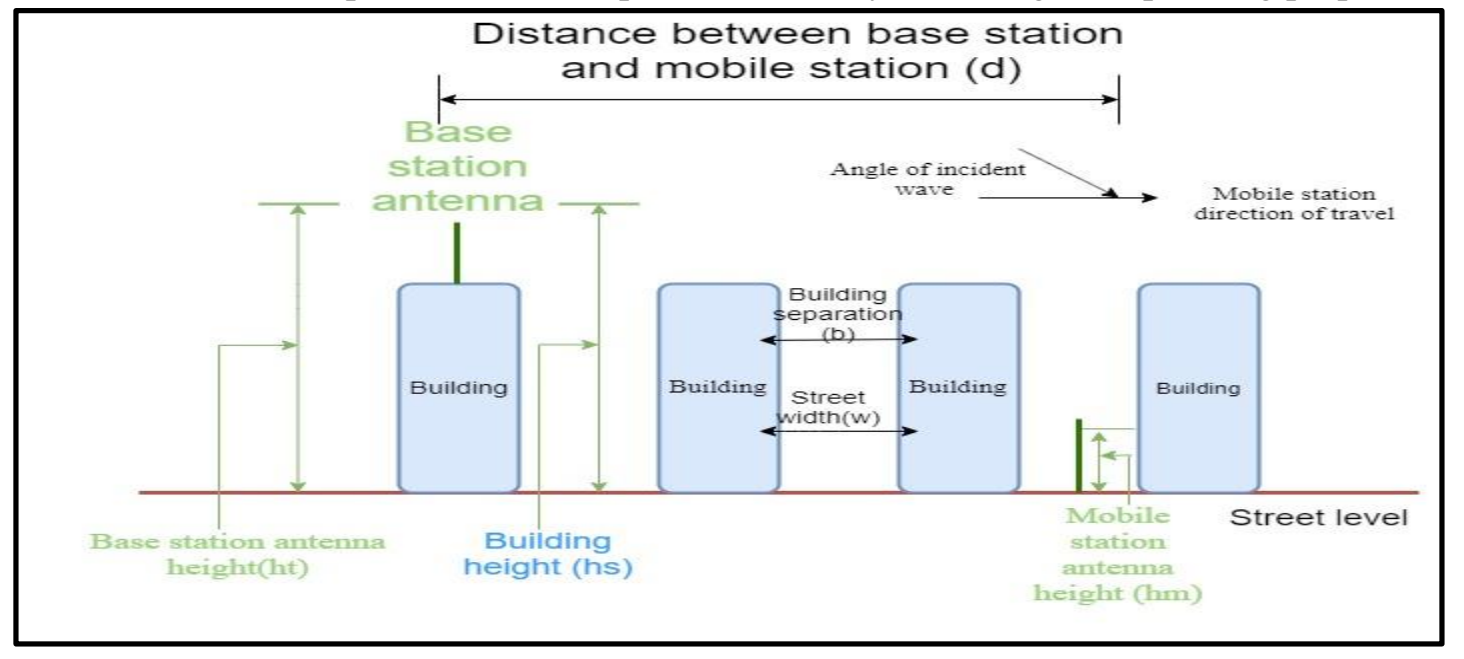

FigURE (2): TYPICAL PATH LOSS VARIABLES AT MOBILE RADIO TELECOMMUNICATION SYSTEM [7].

\section{A. COSTS-231 Hata Mode}

Hata model is an analytical formulation based on the path loss value data raised by Okumura in 1968 in Japan. Hata model is one vastly use paradigms into predicting path loss in a mobile wireless system. This model supplies an expression for medium path loss as a function of the carrier frequency, base station antenna heights, mobile station antenna heights and the distance between BS and MS [8]. It also contains corrections for urban, suburban and rural (flat) environments. The main path loss mathematical equation [5] for this COST-231Hata paradigm can be written as:

$P L(d B)=46.3+33.9 \log _{10} f-13.82 \log _{10} h_{b}+\left(44.9-6.55 \log _{10} h_{b}\right) \log _{10} d-a\left(h_{m}\right)+C_{F} \quad$ eq. $(2)$

Where

* $\mathrm{f}=$ Frequency in $M H_{Z}$

* $h_{b}=$ Height of base station $(M)$ range from (30-200)

* $h_{m}=$ Height of mobile statin $(M)$ range from (1-10)

* $\mathrm{d}=$ Distance from transmitter to the receiver $(\mathrm{Km})$ basically from (1-20)

For urban and suburban areas, $\mathrm{CF}$ is $3 \mathrm{~dB}$ and $0 \mathrm{~dB}$, respectively [4].

In the large cities with high population density urban MS antenna- rectification agent a $\left(h_{m}\right)$ can give by

$a\left(h_{m}\right)=3.20\left[\log _{10}[11.75]\right]^{2}-4.97$

In suburban and rural the MS antenna- rectification agent, a $\left(h_{m}\right)$ can give by

$a\left(h_{m}\right)=\left(1.11 \log _{10} f-0.7\right) h_{m}-\left(1.56 \log _{10} f-0.8\right)$

This model is valid for the following range of parameters [8]:

$>30 \mathrm{~m}<h_{b} \leq 200 \mathrm{~m}$

$>1 \mathrm{~m}<h_{m} \leq 10 \mathrm{~m}$

$>1 \mathrm{~km}<\mathrm{d} \leq 20 \mathrm{~km}$ 


\section{Optimization Approach}

The optimization algorithm is the procedure performed repetitively by comparing various solutions until an optimum result getting. A mathematical model is needed to optimize the design problem[9]. An optimization operation starts by identifying design changes that differs through the operation and placing many limitations of equality and inequality. In that approach, a first step is choosing widely propagation model for predicting path loss in WIMAX network. COST-231 Hata parameters are optimized by genetic algorithm to transform for an appropriate expectation parameters. The optimization target is a minimize path loss predict the best height for base station and mobile station [3].

\section{A. Genetic Algorithm}

Genetic Algorithm (GA) had discovered in the 1960s. It is the research technique that established on basics inspired from by genetic and developmental mechanization present in naturalist systems and groups of living beings [10]. GA supplies success resolutions for a difficult multi-border optimization problem [11],[12],[13] . GA uses to resolve the troubles by an optimal way. The troubles involve detecting the most appropriate solution, finding optimal solutions at an optimization technology. GA acts on a range of potential solutions where each chromosome is represented. Figure (3) shows the basic structure of the GA starter with an initial population of chromosomes. Subsequently, the algorithm goes through an iteration process to make the population evolve till the stopping condition is met and the search stop. Summarize, the main steps for GA consist of the following:-

1. Production of a genetic group randomly from a chromosome.

2. Estimating a fitness $\mathrm{f}(\mathrm{x})$ of every chromosome $\mathrm{x}$ at a population.

3. Create a new community using the following:-

* Choosing two origin chromosomes to create their fitness.

* Formation of new offspring by of the crossover from parents, the admittance from new offspring at a population.

* Change from newly created population to the sum from algorithm.

* end while a preferable solution is returned [13].

\section{B. Materials and Methods}

WIMAX supports broadband covering for high-density urban area and medium-density sub-urban area with an economic infrastructure. The factor that coverage based on is path loss. Therefore, in this paper optimization by GA depend on one of path loss model is COST-231 Hata that widely used wireless network. In this work, the area is divided into urban and sub-urban to determine the best $h_{b}$ and $h_{m}$ with minimal path loss value. The frequency $3.5 G H_{z}$ and distance between $\left(7 k_{m}\right)$ are applied. The results can apply on a planning program such as ICS telecom software a simulator to radio design for better coverage.

\section{Optimization by Genetic Algorithm}

Optimization is done through MATLAB R2015a implementation pack utilizing GA operations at $3.5 \mathrm{GH}_{z}$ with path loss parameters. This implementation is obtaining the worth to the paradigm factors. That is a pact for the area Mensuration best to futurity evolution. G.A is performed by using the fitness function and calculates an average of a difference among the evaluated and measured path loss rates in all evaluation stage in the algorithm. COST-231 Hata uses to a definition from optimized path loss by G.A such as estimates the genomes in its population. The parameters are used in Table1 for the operators of G.A.

Received 11 June 2019; Accepted 8 October 2019 


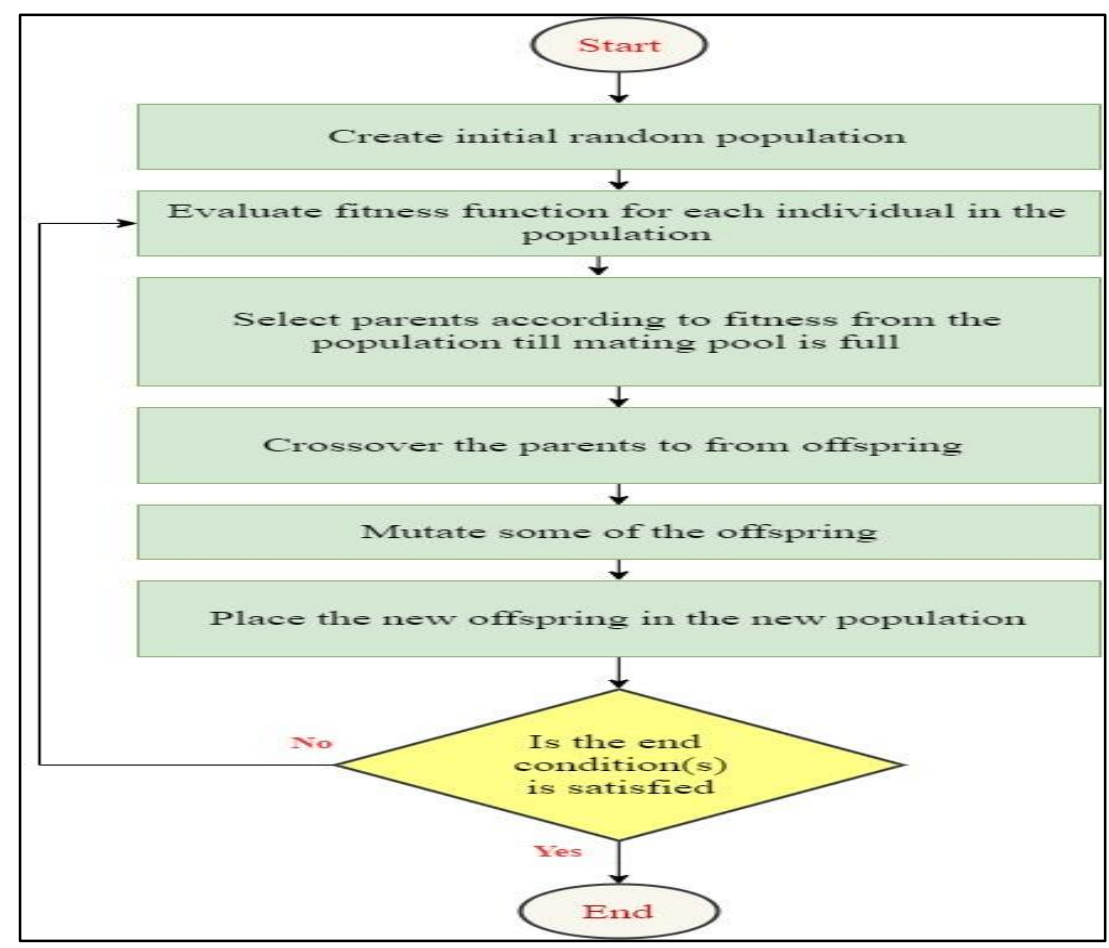

Figure (3) Flowchart of The Genetic Algorithm [4]

TABLE1:- THE PARAMETERS OF GA

\begin{tabular}{|c|c|}
\hline Parameters Group & Value \\
\hline Population Size (N) & 50 \\
\hline Number of Iterations & 100 \\
\hline Crossover rate (Pc) & 2 \\
\hline Mutation rate (Pm) & 1 \\
\hline
\end{tabular}

\section{Results and Discussion}

The results are obtained through the optimization tool GA. GA runs to find a good solution for the path loss. For our simulation, the operating frequency is $3.5 \mathrm{GHz}$ which is a licensed frequency WIMAX a rule in Asian regions. The distance is $(7 \mathrm{~km})$. The other parameters are used shown in table (2). COST-231 Hata parameters are valid for the standard range selected by optimization the path loss by a genetic algorithm as shown in the Table (2).

TABLE (2):- SiMULATION PARAMETERS

\begin{tabular}{|c|c|}
\hline Parameter & value \\
\hline$h_{b}$ & $\{30 \ldots 60\} \mathrm{m}$ \\
\hline$h_{m}$ & $\{1 \ldots .10\} \mathrm{m}$ \\
\hline
\end{tabular}




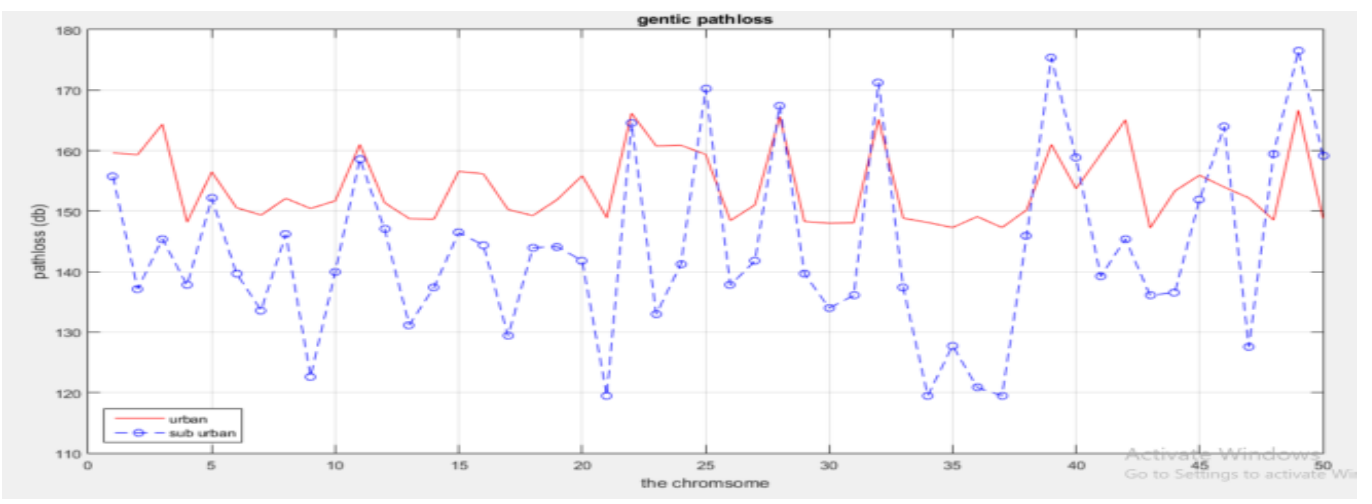

Figure (4) Path loss Measured at Urban and Suburban Environment at Distance (7KM).

Figure (5) shows the result of best chromosome BS and MS height with path loss measure in (dB) at distance $(7 \mathrm{~km})$ for different environments.

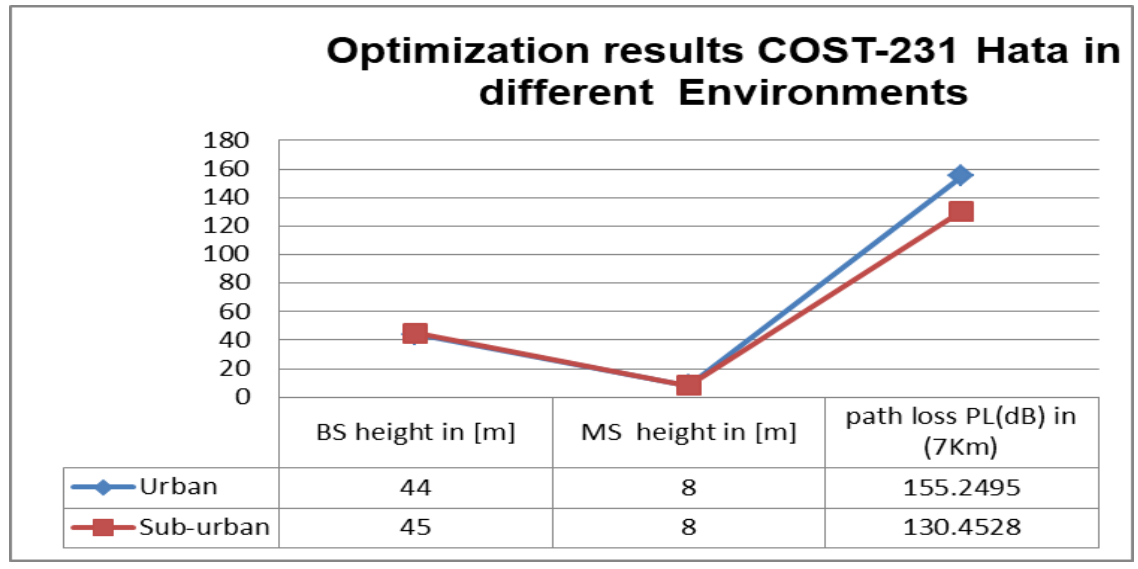

Figure (5) The Best Chromosome For DifFerent Environments IN (7KM)

\section{Conclusion}

In this paper, the path loss value alteration with various environments by the effect of these parameters: $\left(h_{b}, h_{m}\right.$ and $\left.d\right)$. Presently, the COST231-Hata model parameters are used to determine the suitability of the WIMAX network station in different environments namely urban and suburban. The results of the distance simulation are $(3 \mathrm{~km})$ and $(7 \mathrm{~km})$. The comparing optimization results are at different distances. The result of a distance $(3 \mathrm{~km})$ achieve better because of the minimum value in the path loss. The results obtained can be utilized in the Software planning of the wireless system to create a network with better coverage.

\section{REFERANCES}

[1] V. K. Garg, "Wireless communications and networking" San Francisco, Calif.: Morgan Kaufmann, pp.767-768, 2007.

[2] M. Alshami, T. Arslan, J. Thompson, and A. T. Erdogan, "Frequency analysis of path loss models on WIMAX", $3 r d$ Computer Science and Electronic Engineering Conference (CEEC),University of Essex, UK, pp. 1-6, 13-14 July 2011.

[3] M. V. S. N. P. a. P. K. D. Chhaya Dalela, "Tuning Of Cost-231 hata Model for Radio Wave Propagation Predictions "Computer Science \& Information Technology (CS \& IT).Journal, vol. 2, pp. 255-267, 2012.

[4] O. Banimelhem, M. M. Al-Zu'bi, and M. S. A. Salameh, "Hata Path Loss Model Tuning for Cellular Networks in Irbid City", IEEE International Conference on Computer and Information Technology; Ubiquitous Computing and Communications; Dependable, Autonomic and Secure Computing; Pervasive Intelligence and Computing, pp. 1646-1650, 2015.

Received 11 June 2019; Accepted 8 October 2019 
[5] S. M. Hameed, "Indoor Propagation Modeling for Wireless Local Area Network (WLAN) 1", IRAQI JOURNAL OF COMPUTERS, COMMUNICATION AND CONTROL \& SYSTEMS ENGINEERING, vol. 11, pp. 97-105, 2011.

[6] Website Onlin "https://www.avalan.com/blog/bid/324806/indoor-wireless-path-loss-four-factors-that-must-be-considered-fortransmission-range".

[7] R. Saha, "Mobile Wireless Propagation Models and Path Loss Estimation/A Handbook on Cellular Mobile Communication Laboratory A MATLAB-Based Approach": RK Saha, pp.3-9, March 2016.

[8] J. G. Andrews, A. Ghosh, and R. Muhamed, "Fundamentals of WiMAX : understanding broadband wireless networking ". Englewood Cliffs, N.J.: Prentice-Hall,pp.422-424, 2011.

[9] J. A. Snyman, "Practical mathematical optimization: an introduction to basic optimization theory and classical and new gradient-based algorithms". New York: Springer, 2005.

[10] A. Bhuvaneshwari, R. Hemalatha, and T. SatyaSavithri, "Path Loss Model Optimization Using Stochastic Hybrid Genetic Algorithm", International Journal of Engineering and Technology(UAE), vol. 7, pp. 464-469, 10/02 2018.

[11] N. Erradi, N. Aknin, F. Alami, and A. Moussaoui, "Genetic algorithms to optimize base station sitting in WCDMA networks", International Journal of Advanced Computer Science and Applications (IJACSA) vol. 4, pp. 218-220, 04/01 2013.

[12] F. Garzia, C. Perna, and R. Cusani, "Optimization of UMTS Network Planning Using Genetic Algorithms", Communications and Network, vol. 2, pp. 193-199, 01/01 2010.

[13] S. N. Deepa and S. N. Sivanandam," Introduction to genetic algorithms". Berlin: Springer, 2010. 